\title{
Estilos de atribución causal. Importancia para la investigación e intervención profesional en la etapa adolescente \\ Causal Attribution Styles. Importance for Research and Professional Intervention During Adolescence
}

\author{
Carlos Y. Chavarría Carranza \\ Universidad de Costa Rica, Costa Rica
}

\begin{abstract}
Resumen
Se describe el recorrido histórico que ha llevado a lo largo del tiempo la teoría de los estilos de atribución causal hasta llegar al punto que aquí se presenta. Se establecen cercanías y relaciones con otras teorías y paradigmas propios de la Psicología con el fin de ubicarla en este marco general y reconocer en él los particulares aportes que de ella se desprenden. Para ello, se reporta una serie de resultados de investigaciones realizadas donde se muestra no solo la validez de sus constructos, sino también su relación con diversas conductas, actitudes y patologías indagadas. Se discute, además, la importancia de contar con una teoría de este tipo para examinar y, sobre todo, apoyar la intervención psicológica, tanto clínica como social, en la población adolescente.
\end{abstract}

Palabras clave: estilo de atribución causal, internalidad, estabilidad y globalidad, situaciones buenas, situaciones malas, adolescencia

\begin{abstract}
:
In this report, we describe the historical path that led over time to the present-day theory of Causal Attribution Styles. Some similarities and relationships with other theories and paradigms in Psychology are established, in order to acknowledge the place of Causal Attribution Styles in this general framework and identify private contributions. We also discuss the importance of having such a theory to do research and, above all, support intervention in Clinical and Social Psychology, during adolescence. To do this, a number of research findings are reported where they show the validity of their constructs, when they are correlated with other theories, as well as their relationship to various behaviors, attitudes and pathologies of various groups, including adolescent populations.

Keywords: Causal Attribution Style, Internality, Stability and Global, Good Situations, Bad Situations, Adolescence

Carlos Yurán Chavarría Carranza, docente Universidad de Costa Rica, Costa Rica.

La correspondencia en relación con este artículo se dirige a Carlos Yurán Chavarría Carranza, Universidad de Costa Rica, Costa Rica. Dirección electrónica: carlos.chavarria@ucr.ac.cr
\end{abstract}




\section{Y. CHAVARRÍA}

Dicho de forma breve, atribuir es el proceso mediante el cual las personas presumen las causas del comportamiento propio y el de los demás (Rojas, 1992). Por lo tanto, corresponde a un proceso de inferir explicaciones de lo que las personas hacen, por lo que su actuar se constituye en la fuente de información que recae sobre la persona misma "Percibimos a un conductor ejecutando su tarea en forma amable o descortés, no a una persona amable en abstracto" (Martín Baró, 1988, p.206).

Peterson, McClellan y Seligman (1995) señalan que el interés por indagar el estilo de atribución surge en Psicología social en el contexto del debate que generó lo que en su momento se denominó "el control personal". En ese entonces, un área temática importante tenía como eje comprender cómo los pensamientos y las creencias de las personas influyen en sus intentos de controlar los resultados importantes para sus vidas.

Se consideraba por entonces que, dado que las personas difieren entre sí en la forma de dar sentido al mundo, ello llevaba necesariamente a formas distintas de dar significación emocional y motivacional a los eventos del mundo y así en la manera de conducir su actuar en un sentido o en otro.

Estilo de atribución o estilo de explicación es el nombre con el que Peterson et al. (1995) denominan a estas formas diferentes de significación. Se describe como la tendencia de una persona de ofrecer explicaciones semejantes sobre acontecimientos diferentes. De acuerdo con esta definición, los autores señalan lo siguiente:

Podemos identificar un estilo al mirar a través de las diferentes explicaciones; sólo en la medida en que las personas sean coherentes se puede hablar sensatamente que son evidencia de un estilo de explicación. (Peterson et al., 1995, p. 1). (Traducción del suscrito).

Las teorías anteriores, incluso emanadas de paradigmas epistemológicos distintos, se han constituido a la larga en importantes antecedentes para la comprensión de esta acción cognitiva, y así para el desarrollo de la Psicología social.

Según Peterson et al. (1995) se puede encontrar el correlato más añejo en las ideas de Alfred Adler, quien introdujo el concepto de "lucha por la superioridad". En 1912, Adler publicó el texto que denominó "El carácter neurótico" (Adler, 1993), donde señalaba que, desde la infancia, todo individuo experimenta un sentido de fragilidad e inseguridad que define como "sentimiento de inseguridad". Como forma de compensar tal sentimiento, se fijan metas de carácter inconsciente por las cuales la persona se afana en lograr objetivos que le acarreen grados mayores de superioridad.

Según Peterson et al. (1995) ese sentido de superación personal requiere que previamente la persona cuente con alguna creencia de lo que se es y de las propias habilidades. Requiere, por lo tanto, de realizar atribuciones causales sobre sí misma.

Semejantes ideas fueron posteriormente recogidas por los llamados neofreudianos como Erich Fromm, Karen Horney y Harry Sullivan (Peterson et al., 1995), quienes sugerían que la conducta se explica, sobre todo, por situaciones sociales, más que por mecanismos biológicos o instintivos. Estas versiones del psicoanálisis suponen que las personas no responden de forma automática a sus conflictos, más bien lo efectúan de forma activa y creativa. 
ESTILOS DE ATRIBUCIÓN CAUSAL. IMPORTANCIA PARA LA INVESTIGACIÓN E INTERVENCIÓN PROFESIONAL

Erich Fromm se basó en la primera teoría freudiana sobre la organización económica de la libido. En 1932 indicó que la modificabilidad y la plasticidad de los instintos sexuales permite a la persona no solo responder mediante los mecanismos de defensa improductivos, sino también mediante la sublimación de la situación, que implica una actividad personal de tipo consciente para su superación (Fromm, 1972).

Dichas estrategias se desprenderían de las atribuciones causales que realizan las personas sobre las situaciones que deben enfrentar (Peterson et al., 1995). También, afirman que la mayoría de las estrategias de afrontamiento de conflictos, denominados en psicoanálisis como mecanismos de defensa, o más bien todos, pueden entenderse como producto de las subyacentes formas de atribución.

Fritz Heider es reconocido como un importante propulsor de los estudios de atribución (Alvarado \& Garrido, 2003; Martín Baró, 1988; Peterson et al., 1995) y constituyó una base esencial para sus posteriores desarrollos.

Su modelo tiene como fundamento el supuesto de que la persona común, de forma consciente o no, aplica en sus percepciones y en sus comportamientos ciertos presupuestos y principios de cómo las personas son y actúan (Heider, 1944). Heider, además, introdujo asuntos que aún se tratan, como la existencia de explicaciones internas o externas y la corrección o no de ciertas atribuciones (Peterson et al., 1995), que actualmente se consideran como sesgos atribucionales (Alvarado y Garrido, 2003).

A finales de la década de 1950, Heider (citado en Martín Baró, 1988) apuntaba a comprender la vida cotidiana de las personas, o "la psicología ingenua" como la llamó. Según tal perspectiva, no existe alguna importancia real establecer si tales presupuestos o principios resisten al tamiz de alguna prueba de verificación. Lo que se consideraba como central estaba en reconocer las causas fijas internamente a las que se socorre para explicar el comportamiento de los demás. De acuerdo con ello, la persona observada y lo que hace se constituyen en una unidad perceptual, donde la primera (la observada) es la causa de lo segundo.

En la década de 1960, Edward Jones y Keith Davis (citado en Alvarado y Garrido, 2003; Martín Baró, 1988) proponen una variación importante a este modelo de las atribuciones que denominaron de atribución correspondiente. Este modelo se desprende de la teoría de la Gestalt, porque define a la persona observada como la figura y a la situación como el fondo de una unidad completa. De esta forma, quien observa se explicaría lo que una persona $\mathrm{X}$ hace al relacionarla con la situación donde actúa.

Peterson et al. (1995) encuentran una estrecha relación entre las teorías de la atribución y otros desarrollos realizados que, por aquella época de 1960, se estaban produciendo en lo que denominan la "revolución cognitiva". Tal es el caso de la teoría del locus de control de Julian Rotter.

Para Rotter (1966), las conductas están influenciadas por las expectativas de control que cada persona sienta sobre las contingencias de reforzamiento. Las situaciones suceden porque se espera que algo en particular ocurra. Cuando la persona espera que, por lo general, las contingencias de refuerzo de la conducta estén bajo su control, significa que su Locus de Control es Interno (LCI). Contrario a ello, cuando la persona siente que las contingencias de sus actos son producto del azar o del destino su Locus de Control sería Externo (LCE).

Como puede notarse, el estilo de atribución y el locus de control son conceptos que están estrechamente relacionados. Sin embargo, aunque ambos coinciden en establecer la fuente interna o externa en 


\section{Y. CHAVARRÍA}

cuanto a la ubicación de las contingencias de reforzamiento de las conductas, el locus de control parece depender del estilo de atribución de la persona. Según Peterson et al. (1995), en la propia ontogénesis, para que la persona pueda ubicar dentro o fuera de sí el control de las contingencias de reforzamiento, requiere primero que exista una autodefinición sobre la cual se sustente tal ubicación.

Lo anterior se podría apreciar con más claridad en la descripción que diera George Mead (1972) en cuanto a la constitución ontogenética de cada uno. Según él, en toda persona el "Mî" (la persona en cuanto objeto para los demás) aparece primero que el "Yo" (reacción personal frente a los otros). Si consideramos que la persona, de forma reflexiva, realiza atribuciones sobre sí misma de manera semejante a como lo hace con otras personas, ello significa que el control sobre las contingencias, sea externo o interno, se dará como producto de su propia autodefinición. Así, el locus de control estaría determinado por el estilo de atribución característico de cada persona (Peterson et al., 1995).

Además, la teoría del locus de control, tal y como la describiera Rotter (1966), no distingue entre situaciones vividas como positivas o buenas, ni como negativas o malas para la persona y las posibles manifestaciones internas o externas en que se ubiquen las contingencias, según sea cada situación, como sí lo hace la teoría del estilo de atribución, que será tratada más adelante.

Un último e importante constructo teórico, al que nos referiremos, se encuentra en la teoría del aprendizaje social, propuesta por Albert Bandura, específicamente con el concepto de autoeficacia (self-efficacy) (Bandura, 1977). La Autoeficacia es, en concreto, la creencia de una persona de que la respuesta que lleva a un resultado deseado puede ser emitida efectivamente por ella (Bandura, 1977).

También, aquí se observa la relación de este concepto con el estilo de atribución, porque la autoeficacia aumentaría o disminuiría en relación con las atribuciones que, sobre sí misma, haga la persona. Además, de acuerdo con Peterson et al. (1995) ambas teorías se diferencian fundamentalmente en que, mientras la Autoeficacia debe ubicarse en una situación específica, el estilo de atribución es más bien un rasgo persistente en la diversidad de situaciones que viva la persona.

\section{La propuesta de Martin E.P. Seligman sobre el estilo de atribución causal}

En los albores de los estudios de atribución causal, el doctor Martín E.P. Seligman puso en juego este constructo como complemento a otro que él mismo había denominado previamente como indefensión aprendida (Peterson, 1991a).

En congruencia con lo que estipula el paradigma del control de contingencias, se hipotetizaba que cuando la persona no tiene establecida la relación entre conducta y contingencia, lo que quiere decir que no espera un resultado concreto a partir de la emisión de una conducta, se generan en ella una variedad de déficits emocionales, cognitivos y motivacionales (Peterson et al., 1995). A estos déficits se les denomina indefensión aprendida (learned helplessness).

Los déficits aluden a una suerte de desbalance u orientación contradictoria que se genera en la base de cada uno de esos elementos (emociones, cogniciones y motivaciones) y en la relación que se da entre 
ESTILOS DE ATRIBUCIÓN CAUSAL. IMPORTANCIA PARA LA INVESTIGACIÓN E INTERVENCIÓN PROFESIONAL

ellos a la hora de que un organismo se ve impelido a emitir una conducta. En este sentido, los déficits no permiten la asociación conducta - contingencia de modo predecible, lo que imposibilita de modo sensible la emisión de conductas asentadas en motivaciones claramente determinadas.

Seligman, además, asoció en sus estudios sobre indefensión aprendida con la depresión reactiva, porque, para él, ambas compartían características importantes como causas, síntomas, consecuencias, tratamientos y formas de prevención (Peterson et al., 1995).

Aunque el estudio con animales, como los de Landgraf, Long, Der-Avakian, Streets y Welsh. (2015) con ratones o el de Batsching, Wolf y Heisenberg (2016) con moscas, muestran resultados congruentes con lo esperado de acuerdo al modelo teórico, los estudios en seres humanos fueron más dramáticos y variados ya que a veces la indefensión era inducida cuando las personas se habían expuesto durante largo tiempo a situaciones incontrolables para ellas, pero otra veces no. Iguales inconsistencias se daban cuando se exponía a las personas a situaciones distintas, o en cuanto a los efectos diferenciados que se generaban en aspectos como la autoestima (Winefield, 1982; Young y Allin, 1985; Peterson, 1991-a), sólo por señalar algunos casos.

En ese contexto, se empezó a evidenciar que la indefensión aprendida debía guardar alguna relación con la forma en que las personas atribuían lo que les acontecía como consecuencia de emitir una conducta, dado que hay personas que, por ejemplo, revierten lo que les sucede en una situación y, por así decirlo, la irradian a otro tipo de situaciones que, en principio, están desvinculadas, mientras que otras personas no lo hacen. Hay personas que piensan que lo que les sucede, alguna vez, les seguirá aconteciendo a lo largo del tiempo, pero otras no lo piensan así.

Dicho tipo de razonamientos calzaba muy bien con el denominado "modelo de la persona científica", que, según Peterson (1991a), dominó la teoría de las atribuciones causales. Este modelo consideraba tres dimensiones como centrales para ser evaluadas en los estilos de atribución, que son la estabilidad, la globalidad y la internalidad. Peterson et al. (1995) señalan que estas dimensiones fueron tomadas en consideración para proponer un conjunto de hipótesis sobre la forma en que se manifestarían cuando se presenta la indefensión aprendida.

Si la atribución causal es estable, entonces la indefensión inducida es de larga duración; si es inestable, entonces la trasciende. Si la atribución causal es global, entonces la indefensión se manifiesta a través en una variedad de situaciones; si es específica, entonces la indefensión se circunscribe sólo a la situación correspondiente. Por último, si la atribución causal es interna, la autoestima decae; si es externa, la autoestima queda intacta. (Peterson et al., 1995, p.4) (Traducción del investigador).

Los resultados empezaron a mostrar resultados más claros y a verificar la asociación entre ambos constructos. Sin embargo, puede notarse que el modelo se complejiza de manera importante, porque debía indagarse con mayor precisión la forma en que aquellas tres variables (estabilidad, la globalidad y la internalidad) se asocian con la aparición de la indefensión y cuál sería el comportamiento que les "inmunizaría" de ello.

Cuando una persona tiene un estilo de atribución no solo se trata de una situación específica, sino también muestra ser ella misma en distintas y variadas situaciones y mantenerse así en el tiempo. Sin embargo, en los estudios embrionarios esto parecía dejarse de lado. 


\section{Y. CHAVARRÍA}

Un importante sesgo que se muestra con claridad en estas hipótesis iniciales es el que señala Peterson (1991a, 1991b) al referir que los estudios sobre la relación entre la indefensión aprendida y los estilos de atribución fueron realizados fundamentalmente en situaciones negativas. Por eso, cuando la situación se atribuye a causas internas, la autoestima decae, por ejemplo.

Cuando los autores aluden a situaciones buenas o malas no refieren simples apreciaciones éticas para definirlas. En realidad, apuntan al marco de valoraciones con las que una persona clasifica las situaciones que vive. Aunque los reportes de investigación basados en esta teoría suelen usar estos términos, en adelante nos referiremos solo por situaciones positivas o negativas para no generar distracciones innecesarias.

Teniendo eso claro nos preguntamos, ¿qué sucede con el estilo de atribución si la situación experimentada es valorada de forma positiva?

Si se aspiraba a contar con una teoría que llevara a modelos más integrales de comprensión sobre los estilos de atribución, entonces se hacía necesario considerar su eventual dinámica en el marco de las situaciones positivas.

El asunto es claro. Si nos hacemos la siguiente pregunta: ¿se atribuye de igual forma si el signo de la situación es positivo o negativo para la persona? La respuesta no tiene por qué tener una contestación unívoca. En todo caso, un estilo de atribución debe desprenderse de la forma en que se manifiesta de forma consistente en ambos tipos de situación (Peterson, 1991a).

Más intrigante, y a la larga útil según Peterson (1991a), es que tampoco se puede desconsiderar la posibilidad de que el estilo de atribución en situaciones positivas pueda tener un origen y una naturaleza distinta e independiente con respecto a las situaciones negativas. Pero, no hemos logrado reconocer ningún tipo de esfuerzo realizado en ese sentido como para poder reportarlo en este trabajo por lo que solamente lo mencionamos.

Entonces, el estilo insidioso de atribución, como se denomina la contribución por el doctor Seligman (Peterson et al., 1995), contempla e integra tanto estas tres dimensiones como sus eventuales manifestaciones en situaciones positivas o negativas. En resumen, las tres dimensiones son y refieren a:

A. Internalidad - externalidad. Esta dimensión se refiere a la explicación que da la persona a la situación vivida al referirse, a sí mismo, de la forma: "lo que sucede se debe a mí, o por el contrario es debido a otras personas o circunstancias". Si la atribución sobre situaciones negativas es interna, la autoestima se verá afectada en igual sentido.

B. Estabilidad - inestabilidad. En esta dimensión, se establece si la causa de que un evento se presente perdure o no en el tiempo. Se expresa de la forma: "esto se mantendrá en el tiempo o solo se dio así en esta situación". La importancia de esta dimensión radica en que si se hacen las atribuciones a causas estables en situaciones negativas, se tenderá a que en la persona se produzca un déficit crónico de su actividad.

C. Globalidad - especificidad. Se define si la causa de la situación afecta a un abanico de situaciones o solo a una de la siguiente forma: "lo mismo sucederá en otras situaciones de la vida o sólo en esta". La importancia de esta dimensión radica en que si se hacen las atribuciones a causas 
ESTILOS DE ATRIBUCIÓN CAUSAL. IMPORTANCIA PARA LA INVESTIGACIÓN E INTERVENCIÓN PROFESIONAL

globales en situaciones negativas, se tenderá a que en la persona aquellos déficits se generalicen a otras y distintas situaciones.

Como hemos dicho, déficit no significa pasividad. Reiteramos que ello implica una inconsistencia $y$, al final, una carencia de los recursos cognitivos, emocionales y motivacionales centrales para la acción, la resolución de conflictos, afrontar situaciones dilemáticas o de incertidumbre y tomar decisiones.

Estudios como el de Ramírez, Maldonado y Martos (1992) usaron la teoría de esta forma y la pusieron a prueba en relación con la indefensión aprendida para tratar de encontrar tanto el comportamiento de las tres dimensiones que producen que la indefensión se genere, tal como el comportamiento que "inmuniza" a la persona de padecerla. Dos hallazgos importantes de este estudio revelan lo siguiente:

- La inmunización se debilita cuando las personas hacen atribuciones internas y globales sobre eventos negativos.

- La inmunización se fortalece cuando, ante tales eventos, las atribuciones son externas y específicas.

Atribuir los eventos negativos a sí mismo supone que ello se revierte a las distintas situaciones de la vida. Son, entonces, déficits que, al final, desprotegen a la persona de la posibilidad de experimentar la indefensión aprendida.

\section{Los componentes negativos $(\mathrm{CN})$ y positivos $(\mathrm{CP})$ del estilo atribucional}

Como supra se dijo, los estudios sobre la atribución causal estuvieron estrechamente asociados a los de la depresión reactiva debido a las similitudes tanto en forma como en dinámica. Por ello, tanto el doctor Seligman como la investigadora Karen Reivich de la Universidad de Pennsylvania (Reivich, 1995), quien revisa y evalúa las propiedades psicométricas del instrumento que el doctor Seligman diseñó para la evaluación del constructo, proponen agrupar las tres dimensiones en situaciones positivas, por un lado, y las tres dimensiones en situaciones negativas, por el otro. De tales agrupaciones, se obtendrían dos componentes: uno positivo $(\mathrm{CP})$ y otro negativo $(\mathrm{CN})$.

Pues bien, de la relación que se diera entre ambos productos se obtendría un solo indicador global (CP-CN), el cual, señalan ambos autores, fungiría como un importante descriptor de los estados depresivos.

En mucha de la evidencia empírica, se verifica la validez de estos indicadores agrupados. Anderson, Bailey y Sweeney (1986) realizan un metanálisis de 104 estudios que tenían como objeto establecer la relación entre los estilos de atribución y la depresión. En conjunto, estos estudios evalúan a cerca de 15 000 sujetos en cuanto a la relación entre el estilo de atribución y la depresión.

En todos los estudios, se usó el Cuestionario de Estilo Atribucional (Attributional Style Questionnaire, ASQ) construido por el doctor Seligman. Asimismo, los hallazgos permiten evidenciar una importante relación entre depresión y cierto patrón en el estilo de atribución. Cuando en los eventos negativos $(\mathrm{CN})$ las atribuciones tienden a ser internas, globales y estables, la asociación con puntajes altos en depresión suele ser significativa. 


\section{Y. CHAVARRÍA}

De igual manera, cuando los eventos positivos (CP) son atribuidos a causas externas, inestables y específicas, encuentran correlaciones significativas con altos puntajes en depresión. Por lo tanto, se puede notar la correspondencia teórica con las indagaciones empíricas a la hora de construir y verificar hipótesis de investigación.

En otro estudio realizado por Lysaker, Lancaster, Nees y Davis (2004) se indagó el estilo de atribución en 40 pacientes diagnosticados con esquizofrenia. Trataban de ampliar con evidencia empírica la discusión sobre el papel que cumple el estilo de atribución a nivel social, sobre todo, en cuanto al comportamiento disfuncional que evidencian estas personas.

Se aplicó a los participantes el ASQ en una fase posaguda, además de una escala de calidad de vida. Los resultados sugirieron que la tendencia a ver acontecimientos de la vida como resultado de causas inestables o impredecibles se asocian con una mayor disfunción social. Indicaron, además, que la falta de síntomas negativos y la tendencia a hacer atribuciones estables para eventos de la vida, en general, predicen más la presencia de frecuentes contactos sociales, una mayor calidad de la interacción social y mayor participación en la comunidad.

Estos resultados dan cuenta de la importancia de efectuar las agrupaciones en los componentes positivos y negativos, cuando de hacer análisis integrales desde una perspectiva psicosocial se trate.

\section{Importancia de los estilos de atribución causal en la adolescencia. Evidencias empíricas}

Ya sea que se trate de tareas evolutivas (Havinghurst, 1992; Krauskopf, 1980), crisis psicosociales (Erikson, 1974), o reedición de conflictos inconscientes (Blos, 1981; Freud, 1981), la adolescencia es ciertamente un período de cambios sustanciales en la vida de todo ser humano, que se suscitan primero en el cuerpo, luego en el mundo psíquico e inconsciente y, por último, en las relaciones con el mundo y con los demás.

El psicólogo cognoscitivista Jean Piaget (Piaget, 1983) sitúa a eso de los doce años el brote de una nueva forma de pensamiento denominado por él pensamiento abstracto, que se funda sobre el pensamiento concreto propio de la segunda infancia.

El pensamiento abstracto lleva al adolescente a una nueva forma de egocentrismo mediante la creencia íntima en "el poder de la reflexión". Es un tipo de pensamiento hipotético-deductivo, porque le permite a la persona, por medio de teorías y sistemas, fundar hipótesis y extraer conclusiones sobre el comportamiento de los fenómenos particulares del mundo.

Decimos, entonces, que tal habilidad cognitiva facilita un mayor grado de independencia para realizar atribuciones causales sobre lo que acontece a su alrededor. Sin embargo, en gran parte del recorrido por esta etapa, el adolescente no cuenta con los recursos emocionales y cognitivos necesarios para realizar atribuciones equilibradas y suficientemente fundadas. Todo lo contrario.

Como señalara Kurt Lewin (Lewin, 1988), el adolescente es comparable con un tipo de persona marginal y desintegrada del mundo social. El espacio vital del adolescente no se circunscribe a estas 
ESTILOS DE ATRIBUCIÓN CAUSAL. IMPORTANCIA PARA LA INVESTIGACIÓN E INTERVENCIÓN PROFESIONAL

edades ni en el mundo infantil, ni en el mundo adulto. Los cambios que se suscitan en su cuerpo lo locomocionan hacia regiones cognoscitivamente desestructuradas; por lo tanto, se impondrá la tarea de reconfigurarlas y articularlas en un nuevo orden o espacio vital.

Por ello, el adolescente requiere incrementar un sentido de competencia personal. Como dice el psicólogo Robert W. White (citado en Yelon, 1989), una persona es competente cuando “... escoge para su tratamiento continuo aquellos aspectos de su ambiente que ve que es posible afectar en alguna forma. Su conducta es selectiva, dirigida, persistente, en fin, motivada" (p.319).

Debido a que el sentido de competencia requiere de que el pensamiento abstracto esté más desarrollado, pensamos que la teoría de las atribuciones causales, tanto para la indagación como para la intervención profesional, debe emplearse cuando se tenga garantía de que ello es una realidad, es decir a eso de los 15 o 17 años (Moraleda, 1999), o incluso algún tiempo después, cuando la capacidad de intraversión Yoica se pueda ejercitar plenamente. Comprenderían por tanto las etapas que Blos (1981) denomina: adolescencia propiamente, adolescencia tardía y en adelante todo el período post adolescencia.

Es aquí donde cobra especial relevancia la comprensión de las atribuciones causales que muestren a los adolescentes. La adolescencia es un período central en la vida de las personas para fundar aquel sentido de competencia personal que le guiará el resto de su vida. Pero, para que ello se logre de manera satisfactoria, el adolescente deberá, previamente, contar con un estilo de atribución causal que lo faculte en este ejercicio. En este sentido, señala Krauskopf (2002):

Dado que la fase juvenil es un momento clave para redireccionar situaciones de vida que no se podían modificar en el contexto de la dependencia infantil, es un terreno fértil para fomentar mejores estrategias de resolución de problemas. Esto implica abrir nuevas relaciones y espacios que permitan y refuercen el incremento de la capacidad para resolver de modo constructivo las situaciones de riesgo y daño así como para el aprovechamiento óptimo de las oportunidades considerando la resiliencia, la autoprotección y el enriquecimiento del desarrollo (p.17).

Se trata de que el joven pueda balancear las atribuciones causales que realicen, tanto de los eventos positivos como de los eventos negativos, de forma tal que no las extremen y terminen por constituirse en una suerte de esquematización cognitiva insidiosa (Chavarría, 2009), y a fundar el sesgo del "mundo justo", relacionado con la explicación de que el éxito o el fracaso de cada persona se explica porque... "cada quien tiene lo que se merece" (Echebarría, 1996).

Como producto adherido a lo anterior, si no se propician estilos de atribución causal equilibrados, flexibles y amplios en la consideración de situaciones de las que emanan informaciones contrastantes y hasta contradictorias, se puede llegar a establecer lo que Higgins y Bargh denominan el "efecto de autorreferencia" (citado en Baron \& Byrne, 1998) mediante el cual describen la tendencia mostrada por las personas a brindar más atención y privilegiar la información que interesa a su self.

Este efecto de autorreferencia se constituye en una evidencia de que ya la persona posee un estilo de atribución perfectamente establecido. Como decía Seligman, el estilo de atribución es 


\section{Y. ChaVARría}

insidioso y recurrente sin importar, como se señaló con Heider (1944), si las explicaciones resisten o no la prueba de la mera creencia, para constituirse en dato fehaciente. Además, son más fáciles de reconocer como "estilos", en la medida en que las atribuciones pueden ser evocadas de forma más eficientemente e inmediata.

El problema radica en que las atribuciones sesgadas e insidiosas no contribuyen a que la persona despliegue su potencial de actividad en el mundo y le facilite afrontar las distintas situaciones que le plantea la vida de una manera que le signifique mayores niveles de crecimiento personal, asertividad y competencia para actuar en el entorno, tal como lo indica Krauskopf (2002).

Por el contrario, una vez asentados, los estilos insidiosos de atribución conllevan a la realimentación y reproducción del propio self que la persona ha cimentado. Se constituiría, por tanto, en una suerte de autoestereotipo (Chavarría, 2009). En general, esto hace que la persona tienda a relacionarse con el mundo de modo fijo, poco o nada flexible, como si fuese guiada por las fuerzas de un destino ya establecido.

Algo importante de agregar es que, una vez que se dinamizan en el desempeño cognitivo de la persona, los estilos de atribución contribuyen, a su vez, con los procesos de elaboración (conectar informaciones con otras ya existentes) y de categorización (agrupar en categorías); todo con el fin de apoyar a la consolidación del propio self.

En este sentido, Mahoney (1983) resume algunas experiencias de investigación que han dado como producto importantes hallazgos sobre lo que, en terapia cognitiva, se conoce como la técnica de autoinstrucción. Reconoce la importancia que ancestralmente ha tenido este tema, que se resumiría con la frase de Pelman: "todos los pensamientos, especialmente los pensamientos cargados con sentimientos, tienden a convertirse en acciones" (citado en Mahoney, 1983, p.201).

Meinchenbaum (citado en Mahoney, 1983), siguiendo y ampliando esta línea, logró importantes avances en la condición de pacientes esquizofrénicos al transformar las conversaciones internas de tipo absurdas por conversaciones "coherentes y relevantes".

Consideramos que los estilos de atribución calzan perfectamente con esas formas insidiosas de pensamiento; por lo tanto, podrían ser tratados de esta misma manera.

Diversos estudios en ambiente de clase con jóvenes, quienes manifiestan conductas desviadas y hasta suicidas (Mahoney, 1983), se mostraron menos provisorios, menos capaces de resolver problemas hipotéticos y menos eficientes que otros niños y jóvenes del tipo normal (Mahoney, 1983). En general, son menos capaces de emplear el pensamiento de tipo abstracto instrumental (medios con respecto a fines). En concreto, estas personas muestran importantes déficits cognitivos y emocionales que les dificulta circunscribirse en un ambiente, determinar su rol en él y disponer sus habilidades para lograr sus objetivos.

Recordemos que la persona se autocontrola o no de acuerdo con sus estilos insidiosos de atribución, según expusimos cuando relacionamos este constructo con el de locus de control.

Otros estudios realizados sobre los estilos de atribución causal en adolescentes han mostrado importantes asociaciones con varias conductas extremas y patologías, que regularmente se describen como propias del período adolescente. 
ESTILOS DE ATRIBUCIÓN CAUSAL. IMPORTANCIA PARA LA INVESTIGACIÓN E INTERVENCIÓN PROFESIONAL

Cheng y Furnham (2003) emplearon el ASQ en un estudio correlacional junto con otros 4 instrumentos (Autoestima de Rosemberg, las escalas de Afectividad de Bradburn y dos escalas de Felicidad, la de Gurin y la de Oxford.) Se aplicó este conjunto de instrumentos a una muestra de 88 jóvenes de 20 años, que no tuvieran algún tipo de tratamiento clínico.

Los resultados mostraron que el 18\% de variación sobre la felicidad era explicado por el estilo de atribución en conjunto. Además, evaluaron separadamente los dos componentes (negativos y positivos) de las tres dimensiones que establece el ASQ y lograron establecer que, para esta población, el 55\% de la variación sobre la felicidad era explicado por el factor positivo de la atribución causal.

Un hallazgo adicional, en este estudio, muestra que la autoestima establece relaciones con el estilo de atribución, congruentes con lo que cabría esperar lógicamente con respecto al comportamiento de las tres dimensiones y según el signo positivo o negativo de la situación.

Idénticos resultados encontraron Southall y Roberts (2002) cuando investigaron en adolescentes que presentaban sintomatología depresiva posterior a eventos estresantes y su asociación con la autoestima y el estilo de atribución. Los participantes, que mostraron prioritariamente un "estilo atribucional negativo", correlacionaron, a su vez, con baja autoestima y alta "tensión de vida". La autoestima y el estilo de atribución en conjunto dan evidencia de la vulnerabilidad con la que los jóvenes experimentan los síntomas depresivos.

Tabassan y Grainger (2002) realizaron una investigación con una muestra de estudiantes distribuidos según las siguientes características: con y sin problemas de aprendizaje y diagnosticados o no con déficit atencional. Se indagó en ellos el concepto de sí mismos, como estudiantes, la autoeficacia para el logro académico y el estilo de atribución.

Ambos autores establecieron que los estudiantes con problemas de aprendizaje y déficit de atención con hiperactividad mostraron puntajes bajos en el autoconcepto académico, el estilo de atribución y la autoeficacia académicos. Tales puntajes eran significativamente distintos a los que mostraron los pares que no mostraban problemas de aprendizaje ni déficit de atención.

En el contexto nacional, Rojas (1992) se aboca a la adecuación de las escalas "multiatribucional de causalidad" (multiattributional causality sacale, MMCS) diseñadas por B. Weimer (citado en Rojas, 1992) tendientes a determinar la correlación de los estilos de atribución con el éxito escolar.

Como puede notarse, en este estudio, no se utilizó el ASQ. Sin embargo, es importante este antecedente de investigación por ser el único a nivel nacional que indaga los estilos de atribución, previo a la indagación en la que sí se utilizó y adecuó el ASQ (Chavarría, 2009).

Los participantes fueron estudiantes de III ciclo de enseñanza básica y IV ciclo de enseñanza diversificada. Para la adecuación de ítems, se realizó la consulta a dos muestras independientes de 200 estudiantes cada una, mientras que para las pruebas de validez y confianza se aplicó a una muestra de 397 estudiantes. Se evaluaron, además, las variables importantes para el éxito escolar: aceptación social de los compañeros de grupo para realizar actividades de clase y sociales, expectativas de éxito escolar y la historia de fracaso académico. Además, se vigilaron las variables moderadoras sexo y edad (Chavarría, 2009). 


\section{Y. CHAVARRÍA}

El reporte de resultados de las distintas subescalas muestra índices entre moderados y altos en confiabilidad, incluso mayores a los reportados en las aplicaciones en el instrumento original. Se logra determinar que existen distintos patrones de atribución asociados al éxito y al fracaso en las materias escolares, además de que tales diferencias están a su vez moderadas tanto por sexo como por la edad de la persona (Chavarría, 2009).

En síntesis, creemos que con lo reportado en los estudios citados pueden notarse los importantes hallazgos que revelan las indagaciones que utilizan como base la teoría de los estilos de atribución causal, cuando se han indagado en poblaciones adolescentes, sobre todo, en factores tan importantes como los estados emocionales, las patologías psicológicas y el éxito y/o fracaso académico. Estas asociaciones, además, pueden ser consideradas como factores de riesgo o protectores con respecto a otras conductas, tanto deseables como indeseables, pero que tienen gran impacto para el futuro de la persona.

\section{Balance final}

En el presente esfuerzo hemos querido dar cuenta de la importancia que tiene, para la Psicología social como para la Psicología clínica, la teoría de los estilos de atribución causal al enfatizar los aportes que podemos desprender de ella, tanto para la comprensión de diferentes conductas de importancia social, como las que se basan en estereotipos sociales o para cualquier esfuerzo de intervención en la etapa adolescente en aspectos centrales como los estados depresivos y hasta en la ideación suicida.

Nos concentramos en este espacio de vida debido a que es allí cuando afloran cambios cognitivos y emocionales, también fisio-químicos y corporales, que instrumentan al y a la adolescente a interactuar con el mundo de una forma cualitativamente distinta y novedosa en su historia de vida.

Sin embargo, los destinos que se logren en cada campo del desarrollo humano integral están determinados, en gran medida, por los derroteros que les establezca la vida familiar, social y societaria (Chavarría, 2013). En ese sentido, el estilo de atribución causal puede describirse como una habilidad cognitiva todavía en fase de consolidación a esas edades, pero que deviene central para poder actuar en el mundo de una forma proactiva, motivada y sobre todo, competente.

Las formas esquematizadas de pensamiento, que ut supra discutimos, devienen a la larga en un obstáculo para un sujeto que, por ello mismo, se autoestereotipa, estereotipa a las demás personas y sesga significativamente su representación del mundo. Estas formas insidiosas del pensamiento las podemos encontrar detrás de las actitudes y las conductas que pueden resultar o no consonantes con formas culturales y pacíficas de convivencia (Chavarría, 2009, 2013).

Precisamente, en la adolescencia, estos cambios se suscitan y resulta clave apoyar toda acción que busque implementar formas de atribución causal que permitan diferenciar, con suficiente moderación, lo que se establece desde el entorno y lo que le compete propiamente a la persona. Es decir, que le permita distinguir, valorar y describir una situación de otra, con el fin de conjurar una posible tendencia a la globalizar lo que acontece en la vida a los distintos espacios vitales. Por 
ESTILOS DE ATRIBUCIÓN CAUSAL. IMPORTANCIA PARA LA INVESTIGACIÓN E INTERVENCIÓN PROFESIONAL

último, es necesario que permita separar y distinguir el hoy de lo que vendrá, sin suponer que las condiciones de vida se mantendrán fijas, como si el mundo en sí mismo no cambiara.

Si solo lográramos fomentar mayores indicadores en cuanto a moderación y flexibilización de los estilos de atribución causal en los adolescentes, se tendría como consecuencia un aumento de la competencia personal, de la autoeficacia generalizada y de un actuar autónomo y sensible del ser y hacer de los demás. Actitudes y posiciones ante la vida como las que requiere una cultura de paz (Chavarría, 2009).

Pero, esta consecuencia no es solo teórica. Al lado de la historia sobre la discusión conceptual que desarrollamos sobre esta teoría, se sumó un conjunto de investigaciones que la han puesto a prueba y que aportan una importante garantía de su validez y así de su utilidad para ser considerada como parte de los instrumentos con que pueden contar profesionales en Ciencias Sociales, tanto para la investigación académica como para dirigir con sentido teórico la labor profesional.

A pesar de que se requieren mayores esfuerzos, creemos, con este trabajo, haber dejado evidencia importante sobre las virtudes de la teoría que hemos tratado como para que, en el contexto del quehacer de la comunidad académica, sea considerado como "banderazos de salida" para se haga crecer un diálogo necesario, ya no sobre esta teoría, sino sobre cualquiera otra que lleve a mejorar el conocimiento psicológico y psicosocial de las poblaciones a las que nos debemos y extraiga de ellas los mejores insumos para elevar el listón de la calidad en la intervención profesional.

\section{Referencias}

Adler, A. (1993). El carácter neurótico (2 ed.). Barcelona: Paidós Ibérica.

Alvarado, J., \& Garrido, A. (2003). Psicología Social. Perspectivas psicológicas y sociológicas. Madrid: Mc Graw Hill.

Anderson, K., Bailey, S., \& Sweeney, P. (1986). Attibutional Style in Depressión: A Meta-Analitic Riview. Journal of Personality and Social Psychology. 50 (5), 974-991.

Bandura,A.(1977). Self-efficacy:Towardsaunifyingtheoryofbehavioralchange.PsychologicalReview.80(2), 191-215.

Baron, R., \& Byrne, D. (1998). Psicología Social. España: Prentice Hall.

Batsching, S., Wolf, R., \& Heisenberg. H. (2016). Inescapable Stress Changes Walking Behavior in Flies - Learned Helplessness Revisited. PLOS ONE, 11(11), 1-16, https://doi.org/10.1371/journal.pone.0167066

Blos, P. (1981). Psicoanálisis de la adolescencia. México: Editorial Joaquín Mortiz.

Buchanan, G., \& Seligman, M. (1995). Explanatory Style. New Jersey: Lawrence Erlbaum Associates

Chavarria, C.(2009). Locus de controly selección temática televisiva en estudiantes que acuden a colegios catalogados como violentos de zonas urbanas y rurales. (Tesis de doctorado). Universidad para La Paz, Costa Rica.

Chavarría, C. (2013). Estructura Social y Locus de Control en colegios catalogados como violentos de zonas urbanas y rurales. Evidencias de su relación como insumo para la promoción de una Cultura de Paz. Revista Reflexiones. 92 (1), 77-96. 


\section{Y. ChaVARría}

Cheng, H., \& Furnham, A. (2003). Attributional style and self-esteem as predictors of psychological well being. Counselling Psychology Quarterly, 16(2), 121-130.

Echebarría, Agustín. (1996). Sesgos atribucionales. En: Morales, F.; Moya, M.; Fernández, M.; Marques, J.; Páez, D.; Pérez, J. y Turner, J. (Eds.), Psicología Social (p. 253-268). España: McGraw-Hill.

Erikson, E. (1974). Identidad, juventud y crisis. Buenos Aires: Paidos.

Freud, Sigmund. (1981). Sigmund Freud. Obras completas (Tomo II). Madrid: Biblioteca Nueva.

Fromm, E. (1972). Sobre métodos y objetivos de una Psicología Social analítica. En: Peter, H. (Ed.) Marxismo Psicoanálisis y Sexpol (p. 112-142). Buenos Aires. Granica Editores.

Havinghurst, R. (1992). Desarrollo y ajuste personal y social en la adolescencia. La teoría de las tareas evolutivas. En: Psicología Social de la adolescencia. Organización de Estados Americanos: Documentos. 55-61.

Heider, F. (1944). La percepción social y la causalidad fenoménica. Psychological Review, 51(6), 358-374.

Krauskopf, D. (1980). Adolescencia y educación. San José: UNED.

Krauskopf, D. (2002). Juventud en Riesgo y Violencia. Ponencia presentada para el programa Sociedad sin violencia de Programa de las Naciones Unidas para el Desarrollo: El Salvador.

Landgraf, D., Long, J., Der-Avakian, A., Streets, M., \& Welsh, D. (2015). Dissociation of Learned Helplessness and Fear Conditioning in Mice: A Mouse Model of Depression. PLOS ONE 10(4), 1-17.https://doi.org/10.1371/ journal.pone. 0125892

Lewin, K. (1988). La teoría del campo en la ciencia social. España: Paidos.

Lysaker, P., Lancaster, R., Nees, M., \& Davis, L. (2004). Attributional style and symptoms as predictors of social function in schizophrenia. Journal of Rehabilitation Research \& Development, 41(2), 225-232.

Mahoney, M. (1983). Cognición y modificación de la conducta. México: Trillas.

Martín-Baró, Ignacio. (1988). Acción e ideología. Psicología Social desde Centroamérica. San Salvador: UCA Editores.

Mead, G. (1972). Espíritu, persona y sociedad. Desde el punto de vista del conductismo social. Buenos Aires: Paidós.

Moraleda, M. (1999). Psicología del Desarrollo. México: Alfaomega S.A.

Peterson, C. (1991a). The Meaning and Measurement of Explanatory Style. Psychological Inquiry, 2(1), 1-10.

Peterson, C. (1991b). Further Thoughts on Explanatory Style. Psychological Inquiry, 2(1), 50-57.

Peterson, C.; McClellan, G. \& Seligman, M. (1995). Explanatory Style: History and Evolution of the Field. En: Explanatory Style (pp. 1-20). New Jersey: Lawrence Elrlabaum Associates.

Piaget, J. (1983). Seis estudios en psicología. Barcelona: Gráficas Diamante.

Ramírez, E., Maldonado, A., \& Martos, R. (1992). Attributions Modulate Immunization Against Learned Helplessness in Humans. Journal of Personality and Social Psychology, 62(1),139-146

Revista Costarricense de Psicologìa

ISSN 0257-1439 / ISSNe 1659-2913

2019, Vol. 38, N. ${ }^{\circ} 1$ 
ESTILOS DE ATRIBUCIÓN CAUSAL. IMPORTANCIA PARA LA INVESTIGACIÓN E INTERVENCIÓN PROFESIONAL

Reivich, K. (1995). The Measurement of Explanatory Style. New Jersey: Lawrence Elrlabaum Associates.

Rojas, G. (1992). Elaboración de la escala para atribuciones causales para rendimiento académico y afiliación (BACRA). (Tesis de Maestria). Universidad de Costa Rica, Costa Rica.

Rotter, J. (1966). Generalized expectancies for internal versus external control of reinforcement. Psychological Monographs: General and Applied, 80(1), 1-28.

Southall, D. \& Roberts, J. (2002). Attributional Style and Self-Esteem in Vulnerability to Adolescent Depressive Symptoms Following Life Stress: A 14-Week Prospective Study. Cognitive Therapy and Research, 26(5), $563-579$

Tabassan, W., \& Grainger, J. (2002). Self-concept, attribution style and self-efficacy beliefs of students with learning disabilities whith and without attention deficit hyperactivity disorder. Learning disability quarterly, $25,141-151$.

Winefield, A. (1982). Methodological difficulties in demostrating learned helplessness in humans. The Journal of General Psychology, 107, 255-266.

Yelon, S. (1989). Yelon, S. (1989). La psicología en el aula. México: Trillas

Young, L., \& Allin, J. (1985). Persistence of Learned Helplessness in Human. The Journal of General Psychology, $113(1), 81-88$.

Recibido: 19 de Abril de 2017 Revisión recibida: 28 de Enero de 2019

Aceptado: 20 de Marzo de 2019 


\section{Y. ChaVARría}

\section{Sobre el autor:}

Carlos Yurán Chavarría Carranza es psicólogo social. Obtuvo el grado de licenciatura en Psicología en la Universidad de Costa Rica, de donde también es egresado de la Maestría en Psicología con énfasis en Teoría Psicoanalítica. También, se tituló como doctor en Comunicación y Paz en la Universidad para la Paz de la Organización de las Naciones Unidas. Se desempeña como docente en la Universidad de Costa Rica donde ha dictado cursos para diversas carreras. Ha publicado una serie de obras profesionales y artículos científicos en revistas y libros especializados privilegiando los temas de violencia social, la cultura de paz, los procesos cognitivos y la Psicología de la recepción mass-mediática.

Publicado en línea: 28 de junio de 2019 